https://www.journal-imab-bg.org

Original article

\title{
ENTEROCOCCUS AND ENTEROCOCCUS-LIKE ORGANISMS RECOVERED IN FAECAL SCREEN- ING FOR VANCOMYCIN-RESISTANCE
}

\author{
Hristina Y. Hitkova, Preslava M. Hristova \\ Department of Microbiology, Virology and Medical Genetics, Faculty of Phar- \\ macy, Medical University - Pleven, Bulgaria.
}

\begin{abstract}
Background: Fecal screening of patients for vancomycin-resistant enterococci (VRE) is recommended in an attempt to establish infection control measures. Culture-based methods for VRE detection are widely used, but particular attention should be paid to correct identification of growing isolates. This study is focused on species identification and antimicrobial susceptibility of vancomycin-resistant enterococci and other Gram-positive catalase-negative cocci, recovered from VRE screen cultures.

Materials and methods: A total of 109 immunocompromised patients from University Multiprofile Hospital for Active Treatment "Dr G. Stranski", Pleven were screened for VRE. The determination of cocci was relied on cultural characteristics, manual and automated systems for identification as well as data on antimicrobial sensitivity.

Results: A total of 57 Gram-positive catalase-negative cocci were isolated: $32 \mathrm{VRE}, 11$ vancomycin-susceptible enterococci and 14 enterococcus-like organisms. Colonization with VanA or VanC enterococci was detected in $29.35 \%$ of the patients, with a distinct prevalence of VanC $(23.85 \%)$ over than VanA $(5.50 \%)$. Six enterococci were confirmed as vanA genotype - 5 E. faecium and $1 \mathrm{E}$. gallinarum. All E. faecium isolates expressed high-level resistance to vancomycin (MICs $\geq 256 \mu \mathrm{g} / \mathrm{ml}$ ) and low-level resistance to teicoplanin (MICs: 4.0-6.0 $\mu \mathrm{g} / \mathrm{ml}$ ), whereas a single $E$. gallinarum isolate showed MICs $\geq 256 \mu \mathrm{g} / \mathrm{ml}$ for both glycopeptides. The isolated VanC enterococci (13 E. gallinarum and 13 E. casseliflavus) were susceptible to tested antibiotics and possessed low-level resistance to vancomycin (MICs: 4-12 $\mu \mathrm{g} / \mathrm{ml}$ ). Most of the recovered enterococcus-like organisms were identified as Leuconostoc spp.

In conclusion, both species identification and antimicrobial susceptibility pattern have to be taken into account for distinguishing VRE and other Gram-positive catalase-negative cocci, growing in VRE screen cultures.
\end{abstract}

Keywords: VRE, enterococcus-like organisms, fecal screening,

\section{INTRODUCTION}

Members of genus Enterococcus are well known pathogens, and their ecological niche is the intestinal tract of humans and animals. The treatment of enterococci is problematic because of their intrinsic, low-level resistance to â-lactams and aminoglycosides, as well as acquired resistance to penicillin/ampicilin, ciprofloxacin and high concentration of aminoglycosides [1].

The appearance and emergence of vancomycin-resistant enterococci (VRE) in the late 1980s additionally restricts the therapeutic options. Nowadays, nine phenotypes of glycopeptide resistance in enterococci have been described [2]. Eight of these are the result of acquired resistance (VanA, VanB, VanD, VanE, VanG, VanL, VanM, VanN) and one type (VanC) is a natural resistant.

It has been found that the fecal colonization with VRE plays an important role in spreading of these microorganisms in the hospitals and routine screening cultures are recommended in an attempt to establish infection control measures [3]. Culture-based methods for VRE detection in fecal samples are widely used in microbiology laboratories, but particular attention should be paid to correct identification of growing isolates, especially to those which produce colonies similar to enterococci.

Many studies worldwide reveal different aspects of determination of VRE isolated in fecal screening of hospitalized patients $[3,4,5,6,7]$, but the data for Bulgaria are still scarce. These are dated from 2012 when V. Popova et al. [8] studied VRE carriers among dialysis patients. Little is known in our country about the colonization with VRE, predominant glycopeptide resistance types and sensitivity to antimicrobials. This motivates our team to screen hospitalized patients for VRE with attention focused on species identification and antimicrobial susceptibility of enterococcal isolates. Gram-positive catalase-negative nonenterococal strains were also analyzed.

\section{MATERIALS AND METHODS \\ Patients and specimens}

A total of 109 patients from University Multiprofile Hospital for Active Treatment „Georgi Stranski”, Pleven were screened for VRE in the period November 2017 - June 2018. The majority of them were in the Haemodialysis ward (HD) - 97, and 12 were in the Haematology ward (HT). Stools or rectal swabs specimens were collected from the patients.

Selective media for VRE screening

Collected specimens were cultured onto three chro- 
mogenic media - Briliiance VRE agar (Oxoid, UK), chromID VRE agar (bioMerieux, France), HiCrome VRE agar (HI MEDIA, India), and also into a bile esculin azide broth with vancomycin - BEAV (Liofilchelm, Italy). The antibiotic supplement in the chromogenic media was as defined by the manufacturer ( $8 \mu \mathrm{g}$ Vancomycin), whereas BEAV broth was supplemented with $6 \mu \mathrm{g} / \mathrm{ml}$ Vancomycin. Cultures were incubated at $37^{\circ} \mathrm{C}$ and were examined after 24 and $48 \mathrm{~h}$. BEAV broths with black colour were subcultured onto $5 \%$ blood agar plates (BAP) and CPS agar (bioMerieux, France).

\section{Preliminary identification}

Colonies typical for enterococci and other similar colonies were examined by Gram stain and catalase test. All Gram-positive catalase-negative cocci were tested for pyrrolidonyl arylamidase (PYR), lucine amino peptidase (LAP), the presence of streptococcal D antigen, growth in $6,5 \% \mathrm{NaCl}$ and bile esculin agar. For presumptive differentiation of enterococci to group level, additional tests were used - production of acid from manitol and sorbose, fermentation of methyl-alpha-d-glucopyranoside (MGP), arginine dihydrolase (ADH), motility and pigmentation.

\section{Species identification}

The identification up to species level was performed by RapID STREP Panel (Oxoid, UK) and VITEK 2 compact system (bioMerieux, France) with version 7.01 software using Gram-positive test panel (GP-67 cards). Species identi- fication of VanA and misidentified VanC enterococci were determined by VITEK MS (bioMerieux, France).

\section{Antimicrobial susceptibility testing}

The antimicrobial susceptibility was detected by the disk-diffusion method of Bauer-Kirby with standard antibiotic disks $(B D, U K)$ and by VITEK 2 compact system (bioMerieux, France) with AST P-534 cards. The antimicrobial agents tested were ampicillin (AP), gentamicin (GM), norfloxacin (NOR), ciprofloxacin (CIP), vancomycin (VA), teicoplanin (TEC), daptomycin (DAP), linezolid (LZD) and tigecyclin (TGC). Minimal inhibitory concentrations (MICs) were examined by E-test (Liofilchem, Italy). The results were interpreted according to the recommendations of the performance standard of the European Committee on Antimicrobial Susceptibility Testing (EUCAST), 2018. The vanA gene status was confirmed by PCR using C1000 Touch $^{\mathrm{TM}}$ Thermal Cycler (BioRad).

\section{RESULTS}

Overall 57 Gram-positive catalase-negative cocci were isolated in fecal screening of patients for VRE. The distribution of cocci according to the species identification and glycopeptide resistance is presented in Table 1. The data reveal a circulation of VRE with VanA $(n=6)$ and VanC $(n=26)$ phenotypes. Vancomycin-susceptible enterococci (VSE) and nonenterococci were also detected.

Table 1. Species distribution of 57 Gram-positive catalase-negative cocci, isolated in fecal screening for VRE

\begin{tabular}{|c|c|c|c|c|c|c|c|}
\hline \multicolumn{2}{|c|}{ VanA enterococci } & \multicolumn{2}{|c|}{ VanC enterococci } & \multicolumn{2}{|c|}{ VSE } & \multicolumn{2}{|c|}{$\begin{array}{c}\text { Other Gram-positive catalase- } \\
\text { negative cocci }\end{array}$} \\
\hline Species & $\begin{array}{l}\text { Nr of } \\
\text { isolates }\end{array}$ & Species & $\begin{array}{c}\mathrm{Nr} \text { of } \\
\text { isolates }\end{array}$ & Species & $\begin{array}{l}\mathrm{Nr} \text { of } \\
\text { isolates }\end{array}$ & Species & $\begin{array}{l}\mathrm{Nr} \text { of } \\
\text { isolates }\end{array}$ \\
\hline E. faecium & 5 & E. casseliflavus & 13 & E. faecium & 6 & Leuconostoc spp.* & 8 \\
\hline \multirow[t]{3}{*}{ E. gallinarum } & 1 & E. gallinarum & 13 & E. faecalis & 4 & Pediococcus pentosaceus & 1 \\
\hline & & & & E. hirae & 1 & Lactococcus garvieae & 2 \\
\hline & & & & & & Sreptococcus spp.** & 3 \\
\hline Totally & 6 & Totally & 26 & Totally & 11 & Totally & 14 \\
\hline
\end{tabular}

* Leuconostoc spp.: Leuconostoc psudomesenteroides (4), Leuconostoc mesenteroides (3), Leuconostoc citreum (1)

**Sreptococcus spp.: Sreptococcus sanguinis (1), Sreptococcus mutans (1), Sreptococcus gallolyticus ssp. pasteurianus (1)

Generally, colonization with VanA or VanC enterococci was determined in 32 from 109 patients $(29.35 \%)$, as VanC $(23.85 \%)$ being more prevalent than VanA $(5.50 \%)$. Co-colonization with different VRE was not observed, but combinations of vancomycin-susceptible E. faecium and vancomycin-resistant E. faecium, E. casseliflavus or E. gallinarum were present in three patients.

\section{Detection of VRE with VanA phenotype}

Six VanA enterococci were recovered: 5 E. faecium and 1 E. gallinarum. All vancomycin-resistant E. faecium (VR E. faecium) yielded $\alpha$-haemolytic colonies on BAP, violet colonies after subcultivation onto CPS agar and were correctly identified by VITEK 2 compact. The single $E$. gallinarum isolate was $\alpha$-haemolytic on BAP and also formed violet colonies on CPS agar. It was identified by RapID STREP Panel but misidentified by VITEK 2 compact. The species identification of Van A isolates was confirmed by VITEK MS, and the vanA gene was detected by PCR.

The results of antimicrobial susceptibility testing of VanA enterococci are shown in Table 2. All E. faecium isolates expressed the same antibiotic pattern: high-level resistance to ampicillin, gentamicin, and susceptibility to linezolid, daptomycin, tigecycline. The data concerning susceptibility to glycopeptides reveal high-level resistance of E. faecium isolates to vancomycin (MIC $\geq 256 \mu \mathrm{g} / \mathrm{ml}$ ) and low-level resistance to teicoplanin (MIC: 4.0-6.0 $\mu \mathrm{g}$ / $\mathrm{ml}$ ), whereas E. gallinarum isolates showed high-level resistance to both glycopeptides with MICs $\geq 256 \mu \mathrm{g} / \mathrm{ml}$. 
Table 2. Antimicrobial susceptibility patterns of enterococci with VanA phenotype

\begin{tabular}{|c|l|c|c|c|c|c|c|c|c|}
\hline \multirow{2}{*}{$\begin{array}{c}\text { ISOLATE } \\
\text { No }\end{array}$} & \multirow{2}{*}{ Species } & \multicolumn{9}{|c|}{ MICs $(\boldsymbol{\mu g} / \mathbf{m l})$} \\
\cline { 3 - 10 } & & AP & GM & CIP & VA & TEC & LNZ & DAP & TGC \\
\hline HD 11/17 & E. faecium & $\geq 256$ & $\geq 1024$ & $\geq 32$ & $\geq 256$ & 6 & 1.5 & 1 & 0.064 \\
\hline HD 16/17 & E. faecium & $\geq 256$ & $\geq 1024$ & $\geq 32$ & $\geq 256$ & 6 & 2 & 1 & 0.064 \\
\hline HD 36/17 & E. faecium & $\geq 256$ & $\geq 1024$ & $\geq 32$ & $\geq 256$ & 6 & 2 & 1 & 0.064 \\
\hline HD 47/17 & E. faecium & $\geq 256$ & $\geq 1024$ & $\geq 32$ & $\geq 256$ & 4 & 2 & 1 & 0.064 \\
\hline HD 75/17 & E. faecium & $\geq 256$ & $\geq 1024$ & $\geq 32$ & $\geq 256$ & 4 & 1.5 & 1 & 0.064 \\
\hline HD 31/17 & E. gallinarum & $\geq 256$ & $\geq 1024$ & $\geq 32$ & $\geq 256$ & $\geq 256$ & 2 & 2 & 0.064 \\
\hline
\end{tabular}

Detection of VRE with VanC phenotype

A total of 26 VanC enterococci were isolated: $13 \mathrm{E}$. gallinarum and 13 E. casseliflavus. All these were $\alpha-$ haemolytic on BAP and were grown as green colonies on CPS agar.

The data about the species identification and antimicrobial susceptibility of VanC enterococci are listed in Table 3 and Table 4. Among 26 isolates, 17 (65.4\%) were correctly determined in terms of species level by RapID STREP Panel and VITEK 2 compact. The remaining 9 strains $(34.6 \%)$ were with unconformities in the identification, and VITEK 2 compact identified most of them as $E$. gallinarum/E. casseliflavus. Misidentified isolates were motile, so that production of yellow pigment distinguished E. casseliflavus from E. gallinarum and the definitive species were confirmed by VITEK MS.

Table 3. Species identificatian and antimicrobial susceptibility of E. gallinarum isolates with VanC phenotype

\begin{tabular}{|c|c|c|c|c|c|c|c|c|c|}
\hline \multirow{3}{*}{$\begin{array}{c}\text { ISOLATE } \\
\text { No }\end{array}$} & \multicolumn{3}{|c|}{ Identification systems } & \multicolumn{6}{|c|}{ Diameters of growth inhibition zones $(\mathrm{mm})$} \\
\hline & RapID & VITEK 2 & VITEK & $\mathbf{A P}$ & GM & NOR & VA & TEC & LZD \\
\hline & STREP & compact & MS & 2 & 30 & 10 & 5 & 30 & 10 \\
\hline HD $1 / 17$ & E. casseliflavus & E. gallinarum & E. gallinarum & 16 & 15 & 13 & 8 & 19 & 20 \\
\hline HD 5/17 & E. gallinarum & E. gallinarum & - & 19 & 16 & 11 & 9 & 18 & 24 \\
\hline & & E. gallinarum/ & & & & & & & \\
\hline HD 8/17 & E. casseliflavus & E. casseliflavus & E. gallinarum & 18 & 15 & 12 & 9 & 18 & 24 \\
\hline HD 17/17 & E. gallinarum & E. gallinarum & - & 18 & 14 & 6 & 8 & 18 & 25 \\
\hline HD $45 / 17$ & E. gallinarum & E. gallinarum & - & 16 & 14 & 12 & 9 & 19 & 23 \\
\hline HD 59/17 & E. gallinarum & E. gallinarum & - & 16 & 20 & 18 & 9 & 19 & 21 \\
\hline HD $60 / 17$ & E. gallinarum & E. gallinarum & - & 17 & 14 & 11 & 9 & 18 & 24 \\
\hline & & E. gallinarum/ & & & & & & & \\
\hline HD 69/17 & E. casseliflavus & E. casseliflavus & E. gallinarum & 18 & 16 & 13 & 9 & 18 & 24 \\
\hline HD $72 / 17$ & E. gallinarum & E. gallinarum & - & 18 & 15 & 12 & 9 & 19 & 23 \\
\hline HD 76/17 & E. gallinarum & E. gallinarum & - & 17 & 15 & 12 & 9 & 19 & 23 \\
\hline HD $81 / 17$ & E. gallinarum & E. gallinarum & - & 20 & 18 & 14 & 9 & 19 & 22 \\
\hline & & E. gallinarum/ & & & & & & & \\
\hline HD 87/17 & E. casseliflavus & E. casseliflavus & E. gallinarum & 17 & 16 & 13 & 9 & 19 & 23 \\
\hline HD 91/17 & E. gallinarum & E. gallinarum & - & 17 & 15 & 11 & 9 & 18 & 21 \\
\hline
\end{tabular}


Table 4. Species identificatian and antimicrobial susceptibility of E. casseliflavus isolates with VanC phenotype

\begin{tabular}{|c|c|c|c|c|c|c|c|c|c|}
\hline \multirow[b]{2}{*}{$\begin{array}{c}\text { ISOLATE } \\
\text { No }\end{array}$} & \multicolumn{3}{|c|}{ IDENTIFICATION SYSTEMS } & \multicolumn{6}{|c|}{ Diameters of growth inhibition zones $(\mathrm{mm})$} \\
\hline & $\begin{array}{l}\text { RapID } \\
\text { STREP }\end{array}$ & $\begin{array}{r}\text { VITEK } 2 \\
\text { compact }\end{array}$ & $\begin{array}{c}\text { VITEK } \\
\text { MS }\end{array}$ & $\begin{array}{c}\mathbf{A P} \\
2\end{array}$ & $\begin{array}{c}\text { GM } \\
\mathbf{3 0}\end{array}$ & $\begin{array}{c}\text { NOR } \\
10\end{array}$ & $\begin{array}{c}\text { VA } \\
5\end{array}$ & $\begin{array}{c}\text { TEC } \\
\mathbf{3 0}\end{array}$ & $\begin{array}{c}\text { LZD } \\
10\end{array}$ \\
\hline HD $6 / 17$ & E. casseliflavus & $\begin{array}{l}\text { E. gallinarum/ } \\
\text { E. casseliflavus }\end{array}$ & E. casseliflavus & 20 & 16 & 15 & 10 & 18 & 24 \\
\hline HD 33/17 & E. gallinarum & $\begin{array}{l}\text { E. gallinarum/ } \\
\text { E. casseliflavus }\end{array}$ & E. casseliflavus & 19 & 15 & 14 & 10 & 16 & 21 \\
\hline HD 40/17 & E. casseliflavus & E. casseliflavus & - & 18 & 17 & 15 & 9 & 16 & 21 \\
\hline HD $41 / 17$ & E. casseliflavus & E. casseliflavus & - & 20 & 17 & 16 & 10 & 19 & 24 \\
\hline HD 70/17 & E. gallinarum & $\begin{array}{l}\text { E. gallinarum/ } \\
\text { E. casseliflavus }\end{array}$ & E. casseliflavus & 20 & 17 & 16 & 10 & 19 & 24 \\
\hline HD $84 / 17$ & E. casseliflavus & E. casseliflavus & - & 20 & 16 & 16 & 10 & 19 & 23 \\
\hline HD 93/17 & E. gallinarum & E. casseliflavus & E. casseliflavus & 20 & 16 & 16 & 11 & 19 & 24 \\
\hline HD 96/17 & E. casseliflavus & E. casseliflavus & - & 16 & 16 & 15 & 10 & 18 & 20 \\
\hline HD 99/17 & E. casseliflavus & E. casseliflavus & - & 20 & 19 & 18 & 11 & 19 & 25 \\
\hline HT $4 / 18$ & E. gallinarum & $\begin{array}{l}\text { E. gallinarum/ } \\
\text { E. casseliflavus }\end{array}$ & E. casseliflavus & 20 & 19 & 16 & 11 & 19 & 25 \\
\hline HT 6/18 & E. casseliflavus & E. casseliflavus & - & 22 & 19 & 17 & 11 & 19 & 20 \\
\hline HT 10/18 & E. casseliflavus & E. casseliflavus & - & 22 & 18 & 20 & 11 & 20 & 25 \\
\hline HD $27 / 18$ & E. casseliflavus & E. casseliflavus & - & 20 & 16 & 14 & 11 & 16 & 20 \\
\hline
\end{tabular}

The results of disk-diffusion tests reveal that VanC enterococci were susceptible to ampicillin, gentamicin, teicoplanin, linezolid, and possess reduced susceptibility to vancomycin with a zone of growth inhibition 8-9 $\mathrm{mm}$ and 9-11 $\mathrm{mm}$ for E. gallinarum and E. casseliflavus, respectively. For both species, vancomycin MICs varied from 4 to $12 \mu \mathrm{g} /$ $\mathrm{ml}$, whereas teicoplanin MICs were between 0.5-0.75 $\mu \mathrm{g} / \mathrm{ml}$, which corresponds to VanC phenotype.

Detection of vancomycin susceptible enterococci

Most of isolated VSE belong to the species E. faecium and $E$. faecalis (see Table 1). Two enterococcal species, $E$. faecalis and $E$. hirae, were recovered from one patient. VSE appeared as $\alpha$ - or non-haemolytic on BAP and produced typical turquoise colonies on CPS agar. These cocci were tentatively identified by RapID STREP Panel and/or VITEK 2 compact. Two E. faecalis isolates were determined by both systems as E. gallinarum (HT 1/18) and E. casseliflavus (HD 94/17), but they were nonmotile, nonpigmented, MGP-negative and susceptible to vancomycin. Further testing by VITEK MS confirmed E. faecalis species. Some variations in susceptibility to antimicrobials were observed among VSE isolates, but all were susceptible to glycopeptides (data not shown).

Detection of Gram-positive catalase-negative cocci other then enterococci

A total of 14 enterococcus-like organisms were detected in the fecal screening for VRE (see Table 1). Most of them $(66.28 \%)$ belong to genera Leuconostoc $(n=8)$ and Pediococcus $(\mathrm{n}=1)$, which are naturally resistant to glycopeptides. Isolates of Lactococcus $(n=2)$ and Sreptococcus $(n=3)$ were also present.

Nonenterococcal species produced colonies similar to those of enterococci, but much smaller, $\alpha$ - or non-haemolytic on BAP and variably coloured on CPS agar - green, pink or colourless. All these were Gram-positive cocci, nonmotile, catalase and PYR-negative, with the exception of both strains Lactococcus garvieae (previously named as Enrerococcus seriolicide), which were PYR-positive. Most of these cocci didn't produce acid from manitol and sorbose, and expressed variable results regarding LAP, $\mathrm{ADH}$, bileesculin test, growth in $6,5 \% \mathrm{NaCl}$ and streptococcal D antigen. The definitive identification of non-enterococci, based on VITEK 2 compact system, was as follows: Lactococcus garvieae - 2, Leuconostoc pseudomesenteroides - 4, Leuconostoc mesenteroides -3 and single strains of Leuconostoc citreum, Pediococcus pentosaceus, Streptococcus sanguinis, Streptococcus mutans, Streptococcus gallolyticus ssp pasteurianus. Susceptibility to almost all tested antimicrobials was found in lactococci and streptococci, whereas members of Leuconostoc and Pediococcus were susceptible to ampicillin, gentamicin and linezolid, but resistant to both glycopeptides without a zone of growth inhibition by the disk diffusion method. The values of MIC confirmed highlevel resistance to vancomycin ( $\mathrm{MIC} \geq 256 \mu \mathrm{g} / \mathrm{ml}$ ) and teicoplanin (MIC: 128-256 $\mu \mathrm{g} / \mathrm{ml}$ ).

\section{DISCUSSION}

This study provides information about species identification and antimicrobial susceptibility of intestinal VRE isolated from immunocompromised patients. The data reveal 
that culture-based screening for VRE can detect VSE and nonenterococci, too.

In summary, from a total of 57 recovered Gram-positive catalase-negative cocci, 32 were identified as VRE, 11 as VSE and 14 as nonenterococci. Our data, like proportion, are comparable to those of D.F.J. Brown and E. Walpole [4], who reported $128 \mathrm{VRE}, 36 \mathrm{VSE}$ and organisms other than enterococci in fecal screening for VRE. According to Gordts et al. [5], out of 135 enterococcus-like organisms, 113 (83.8\%) were identified as Leuconostoc spp. or vancomycin- and teicoplanin-susceptible enterococci. Other trials have also revealed detection of non-VRE in culturing of specimens onto vancomycin selective agars and broths $[6,7]$.

All studied cocci were recovered from BEAV broth, but 4 VR E. faecium, 1 E. gallinarum and 1 E. casseliflavus isolates were directly growing onto solid chromogenic media. The presumptive identification of VRE culturing on chromogenic media was based on typical coloured colonies as per manufacturer's instructions. In contrast, the recognition of enterococci growing after subcultivation from BEAV broth onto BAP and CPS agar required many supplemental tests as recommended in diagnostic manuals [9, 10]. Grampositive catalase-negative cocci, which were PYR, LAP and bile esculin positive, possessed streptococcal $\mathrm{D}$ antigen and were grown in $6,5 \% \mathrm{NaCl}$ were determined presumptively as enterococci. Further identification to group level was based on MGP-test, hydrolysis of arginine, production of acid from manitol and sorbose, motility and pigment, but these tests could not fully differentiate the species.

The definitive identification of isolated enterococci was relied on manual and automated systems, but both systems provided some incorrect results. Furthermore, VITEK 2 compact produced results with low discrimination as $E$. gallinarum/E. casseliflavus in about $1 / 4$ of VanC enterococci, but additional tests such as motility and pigment production solved the discrepancies. It is known that the VITEK 2 database cannot reliably differentiate E. gallinarum from E. casseliflavus and presents most of them as E. gallinarum/ E. casseliflavus [11]. According to the publication of $\mathrm{M}$. Abele-Horn et al. [12], VITEK 2 with version 4.01 software had correctly identified 114 of 121 (94.2\%) strains to the species level as E. faecium, E. gallinarum or E. casseliflavus. The VITEK 2 system was used for the identification of VRE in routine microbiology work, but this system is less efficient in detecting non-faecalis and non-faecium species [13] Because of that, isolates with unconformities in the identification were tested with MALDI-TOF (Matrix-assisted Laser Desorption Ionization-Time-of-Flight) technology using VITEK MS (bioMérieux, France), which is a highly accurate metod for identification of enterococci [10].

In the present study, cocci other then enterococci were identified to the genus level on the basis of conventional tests (e.g. PYR, LAP, bile-esculin test, grown in 6,5\% $\mathrm{NaCl}$, streptococcal D antigen), resistance/susceptibility to vancomycin, and furthermore to the species level by VITEK 2 compact. The commercial diagnostic systems can misidentify unusual Gram-positive catalase-negative cocci such as Aerococcus, Leuconostoc, etc., mostly at the species level
$[14,15]$. Conventional phenotypic tests with assistance to genotyping assays (PCR, 16S rDNA sequence analysis, etc.) have been recommended for these purposes [16]. Nowadays, MALDI-TOF MS technology is used successfully for the identification of this group of bacteria [14].

The determination of enterococcal species can somewhat point to the antimicrobial sensitivity, but full susceptibility testing was performed to acquire such data and to determine resistance phenotype. On the other hand, tests for antimicrobial sensitivity can assist in identification [17]. For example, a specific antimicrobial pattern was observed in our Leuconostoc spp. - susceptibility to almost all tested antibiotics and high-level resistance to vancomycin and teicoplanin with MICs similar to those already published [18]. Also, overall 26 E. gallinarum and E. casseliflavus strains were highly sensitive to many antibiotics with the exception of vancomycin - the zone of growth inhibition varied between 8-11 mm and MICs values confirmed lowlevel vancomycin resistance. These data are related to VanC species. The single unusual E. gallinarum strain with highlevel resistance to vancomycin and teicoplanin was confirmed as vanA genotype.

Interestingly, VR E. faecium isolates possessed the same growth characteristics (violet colonies on CPS agar) and multidrug resistant pattern, expressing high-level resistance to vancomycin and low-level resistance to teicoplanin. These results do not fully match to VanA phenotype, which is characterized with high-level resistance to both glycopeptides, but vanA gene was detected. Furthermore, identical phenotypic features, concerning colonial morphology and antimicrobial testing, draw attention to the search of genetic relatedness between the isolates. VR E. faecium isolates with these specific characteristics have been circulating in our hospital since 2013 [19]. The molecular analysis of our previously recovered VR E. faecium $(\mathrm{n}=60)$ has determined vanA gene and confirmed circulation of a single dominant genotype ( $95 \%$ of isolates) grouped into ST203 (CC17) [20].

Our study has some limitations, concerning the absence of precise genetic techniques for characterization of glycopeptide resistance mechanisms, clonal complexes and allelic profile of strains. Further investigations will be performed in this direction.

In conclusion, both species identification and antimicrobial susceptibility pattern have to be taken into account for distinguishing VRE, but advanced technologies (e.g. MALDI-TOF, nucleic acid sequencing, etc.) are necessarily for definitive identifications at species level and determination of antimicrobial resistance mechanisms.

\section{ACKNOWLEDGMENTS}

This study was carried out with the financial support of Medical University - Pleven through University Grants Commission (Project No. 17/2017).

We are grateful to prof. Grazyna Mlynarczyk, $\mathrm{PhD}$ for logistic support and thank Szymon Walter de Walthoffen, $\mathrm{PhD}$ for collaboration in the Department of Medical Microbiology, Warsaw Medical University, Poland. 


\section{REFERENCES:}

1. Murray BE. The life and times of the Enterococcus. Clin Microbiol Rev. 1990 Jan;3(1):46-65. [Crossref]

2. Cattoir V, Leclercq R. Twenty-five years of shared life with vancomycinresistant enterococci: is it time to divorce? J Antimicrob Chemother. 2013 Apr;68(4):731-42. [PubMed] [Crossref]

3. Faron LM, Ledeboer AN, Buchan WB. Resistance mechanisms, epidemiology, and approaches to screening for vancomycin-resistant Enterococcus in the health care setting. J Clin Microbiol. 2016 Oct; 54(10):2436-47. [PubMed] [Crossref]-

4. Brown DFJ, Walpole E. Evaluation of selective and enrichment media for isolation of glycopeptide-resistant enterococci from faecal specimens. $J$ Antimicrob Chemother. 2003 Feb;51(2):289-96. [PubMed]

5. Gordts B, Van Landuyt H, Ieven M, Vandamme P, Goossens H. Vancomycin-resistant enterococci colonizing the intestinal tracts of hospitalized patients. J Clin Microbiol. 1995 Nov;33(11):2842-6. [PubMed]

6. Kallstrom G, Doern CD, Dunne MW. Evaluation of a chromogenic agar under development to screen for VRE colonization. J Clin Microbiol. 2010 Mar;48(3):999-1001. [PubMed]

7.Suwantarat N, Roberts A, Prestridgem J, Seeleym R, Speser S, Harmon C, et al. Comparison of five chromogenic media for recovery of vancomycin-resistant enterococci from fecal samples. J Clin Microbiol. 2014 Nov;52(11):4039-42. [PubMed] [Crossref]

8. Popova V, Sredkova M, Hitkova H, Todorov V, Borisov B, Dimitrova B. Fecal colonization with vancomycinresistant enterococci in haemodialysis patients. XI National Congress on Clinical Microbiology and Infections, Sofia, 9-11.05.2013; p.23.

9. Christensen J, Ruoff K. Aerococcus, Abiotrophia, and other aerobic catalase-negative Gram-positive cocci. In: Manual of clinical microbiology. Jorgensen JH, Pfaller MA, Carroll KC, eds. 11th edition. Washington, DC: ASM Press. 2015. pp. 422-436.

10. Teixeira ML, Carvalho SM, Facklam R, Shewmaker LP. Enterococcus. In: Manual of clinical microbiology. Jorgensen JH, Pfaller MA, Carroll $\mathrm{KC}$, eds. 11th edition. Washington, DC: ASM Press. 2015. pp. 403-421.

11. Ramotar K, Woods W, Larocque L, Toye B. Comparison of phenotypic methods to identify enterococci intrinsically resistant to vancomycin (vanC VRE). Diagn Microbiol Infect Dis. 2000 Feb;36(2): 119-24. [PubMed]

12. Abele-Horn M, Hommers L, Trabold R, Frosch M. Validation of VITEK 2 version 4.01 software for detection,identification, and classification of glycopeptide-resistant enterococci. J Clin Microbiol. 2006 Jan; 44(1):71-76. [PubMed]

13. Fang H, Ohlsson AK, Ullberg M, Ozenci V. Evaluation of species-specific PCR, Bruker MS, VITEK MS and the VITEK 2 system for the identification of clinical Enterococcus isolates. Eur J Clin Microbiol Infect Dis. 2012 Nov;31(11):3073-7. [PubMed]

14. Almuzara M, Barberis C, Velazquez VR, Ramirez MS, Famiglietti A, Vay C. Matrix-assisted Laser Desorption Ionization-Time-of-Flight Mass Spectrometry (MALDI-TOF MS) as a Reliable Tool to Identify Species of Catalase-negative Gram-positive Cocci not Belonging to the Streptococ- cus Genus. Open Microbiol J. 2016 Dec 28;10:202-208. [PubMed] [Crossref]

15. Winston L.G, Pang S, Haller BL, Wong M, Chambers HF 3rd, PerdreauRemington F. API 20 strep identification system may incorrectly speciate enterococci with low level resistance to vancomycin. Diagn Microbiol Infect Dis. 2004 Apr;48(4): 287-8. [PubMed]

16. Kulwichit W, Nilgate S, Chatsuwan T, Krajiw S, Unhasuta C, Chongthaleong A. Accuracies of Leuconostoc phenotypic identification: a comparison of API systems and conventional phenotypic assays. BMC Infect Dis. 2007;7:69-77.

17. Quiloan MLG, Vu J, Carvalho J. Enterococcus faecalis can be distinguished from Enterococcus faecium via differential susceptibility to antibiotics and growth and fermentation characteristics on mannitol salt agar. Front Biol. 2012 Apr;7(2):167-177. [Crossref]

18. Swenson JM, Facklam RR, Thornsberry C. Antimicrobial susceptibility of vancomycin-resistant Leuconostoc, Pediococcus, and Lactobacillus species. J Antimicrob Chemother. 1990;34(4):543-549.

19. Popova V, Hitkova H, Ivanov I, Sredkova M, Biseva L, Edreva V, et al. Emergence of vancomycin-resistant enterococci in University HospitalPleven. XII National Congress on Clinical Microbiology and Infections, Sofia, 24-26.04.2014; p.19.

20. Ivanov IN, Dobrinov V, Hitkova H, Popova V, Ivanova K, Dobreva E, et al. Molecular characterization of a large hospital outbreak caused by glycopeptide-resistant Enterococcus faecium ST203(CC17). 28th ECCMID, Madrid, Spain, 21-24.04.2018, PO 460.

Please cite this article as: Hitkova HY, Hristova PM. Enterococcus and Enterococcus-like organisms recovered in faecal screening for vancomycin-resistance. J of IMAB. 2019 Jul-Sep;25(3):2649-2654.

DOI: https://doi.org/10.5272/jimab.2019253.2649

Received: 30/11/2018; Published online: 05/08/2019

\section{Address for correspondence:}

Hristina Y. Hitkova, PhD; Department of Microbiology, Virology and Medical Genetics, Medical University - Pleven

1, St. Kliment Ohridski str., 5800 Pleven, Bulgaria

E-mail: hitkova@abv.bg 